\title{
SÍNDROME DE MOEBIUS
}

\section{Relato de caso}

\author{
Lucia Fontenelle', Alexandra Prufer de Q.C. Araujo', Rosiane S. Fontana ${ }^{2}$
}

\begin{abstract}
RESUMO - Nos últimos anos, temos tido oportunidade de diagnosticar maior número de casos da síndrome de Moebius, possivelmente como resultado de aumento real da sua incidência - já que numerosos fatores ambientais vêm sendo relacionados à gênese dessa síndrome. Dos casos avaliados em dois ambulatórios especializados, vale a pena registrar um deles devido à associação incomum com heterotopia cortical, dentre outras malformações.
\end{abstract}

PALAVRAS-CHAVES: síndrome de Moebius, paralisias de nervos cranianos, malformações congênitas.

Moebius syndrome: case report

ABSTRACT - In the last few years we have been able to diagnose a larger number of cases of Moebius syndrome, perhaps due to its increased incidence - since a number of environmental factors have been related to the genesis of the syndrome. Out of the cases evaluated in two specialized ambulatory, it is worthwhile to mention one of them due to an uncommon association with cortical heterotopy, among others malformations.

KEY WORDS: Moebius' syndrome, cranial nerves paralysis, congenital malformation.

A síndrome de Moebius (SM) foi descrita por Moebius ${ }^{1}$, em 1892. Caracteriza-se por paralisia congênita e não progressiva do VII e do VI nervos cranianos (NC), quase sempre bilateral, o que produz uma aparência facial pouco expressiva e estrabismo convergente. Frequentemente outros NC apresentam-se comprometidos, uni ou bilateralmente, determinando ptose palpebral, estrabismo divergente, surdez, distúrbios da sensibilidade nos territórios inervados pelo trigêmeo, disfagia, disfonia e atrofia da língua, que podem ser verificados em diferentes combinações. Número significativo de casos acompanha-se de deficiência mental sugerindo que o envolvimento do sistema nervoso possa ser mais difuso do que o apontado pela presença maciça das paralisias de NC. Malformações esqueléticas estão presentes em grande número de casos, especialmente pés tortos. Micrognatia e aplasia do peitoral também são observadas em associação à SM, constituíndo a denominada síndrome de Polland ${ }^{2}$. Já foram descritas outras anormalidades, como defeitos das extremidades, dos dentes ${ }^{3}$, $\operatorname{cardíacos}^{4}$ e disfunção respiratória central ${ }^{5-7}$, dentre outras menos comuns.
A origem da SM permanece especulativa mas, certamente, é influenciada por fatores genéticos e ambientais. Isquemia fetal transitória é a teoria mais aceita para explicar a síndrome ${ }^{8,9}$. Segundo seus defensores, qualquer alteração que prejudique o fluxo sanguíneo da placenta para o feto, num certo momento da gestação, poderia originar a aplasia ou a hipoplasia dos núcleos dos nervos facial e motor ocular externo, no tronco cerebral. Embora a maioria dos casos seja esporádica, alguns estudiosos postulam a natureza genética para a SM: dentre eles, Dotti e col. ${ }^{8}$ descreveram evidências de herança dominante em duas famílias e Ziter e col. ${ }^{10}$ reconheceram que a translocação entre o braço curto do cromossoma 1 e o braço longo do 13 era responsável por casos de SM na mesma família. Independentemente da teoria principal, alguns fatores ambientais têm sido implicados na gênese da SM: hipertermia, exposição da gestante a infecção, utilização do misoprostol, do álcool, da cocaína, da talidomida e de benzodiazepínicos, entre outros. Acreditamos que a popularização do uso do misoprostol como abortivo, em nosso meio, possa ser responsável pelo au-

\footnotetext{
Instituto de Puericultura e de Pediatria Martagão Gesteira da Universidade Federal do Rio de Janeiro (IPPMG-UFRJ) e Hospital Municipal Miguel Couto (HMMC): ${ }^{1}$ Professora-Adjunta da Faculdade de Medicina da UFRJ, Neuropediatra do IPPMG e do HMMC; ${ }^{2}$ Residente de Pediatria, com especialização em Neuropediatria do IPPMG-UFRJ, pediatra do HMMC.
} 
mento do número de casos da SM observado nos anos recentes. Estudos realizados em nosso país s,11 $^{\text {têm }}$ deixado evidente essa relação. No período de 4 e meio anos (entre junho de 1996 e janeiro de 2001) tivemos a oportunidade de diagnosticar nove casos da SM: seis no Instituto de Puericultura e Pediatria Martagão Gesteira da Universidade Federal do Rio de Janeiro (IPPMG) e três casos no Hospital Municipal Miguel Couto da Secretaria da Saúde da cidade do Rio de Janeiro (HMMC). Dentre tais casos, nos chamou a atenção o que será relatado a seguir, face à presença de heterotopia cortical, cuja descrição anterior desconhecemos. A publicação do caso e das fotos foi autorizada e assinada pela mãe do paciente, tendo sido o termo de consentimento anexado ao prontuário hospitalar.

\section{CASO}

Menino, nascido em 19/12/1995, branco, natural do Rio de Janeiro, foi atendido no Ambulatório de Neuropediatria do IPPMG, pela primeira vez em 13/6/1996, devido a crises convulsivas febris recorrentes. Era o quarto filho de $5^{a}$ gestação, sem intercorrências. Havia relato de um

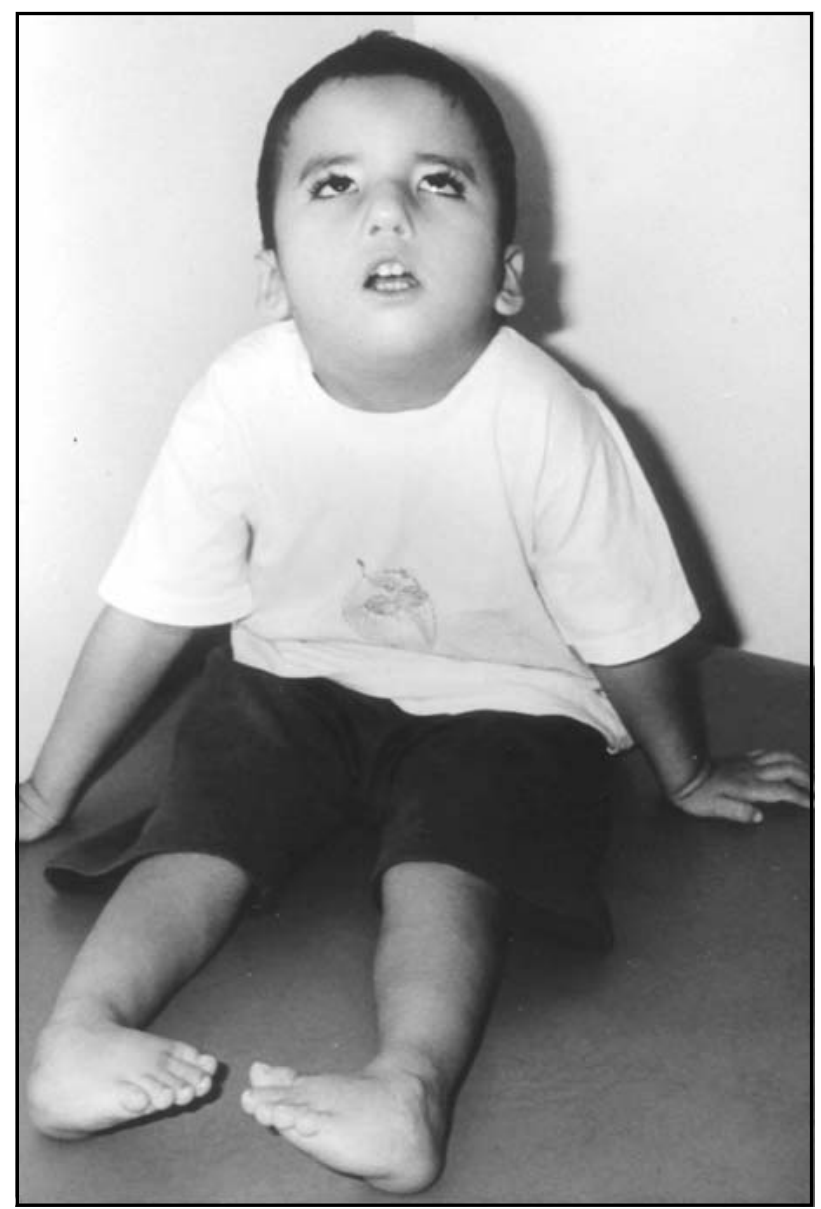

Fig 1. Paciente aos 3 anos de idade. Observa-se as características faciais e os pés tortos. (Foto publicada com a autorização escrita da mãe do paciente).
Tabela 1. Resultados dos exames complementares de diagnóstico.

\begin{tabular}{ll}
\hline US cerebral & Leve aumento das cavidades ventriculares \\
US abdominal & Normal \\
EEG & Paroxismos de pontas de 4 a 5 ciclos/seg \\
Pot. Evoc. Auditivo & Normal \\
Cariotipo & $46 \mathrm{XY}$ \\
Fundo de olho & Normal \\
\hline
\end{tabular}

US, ultra-sonografia; EEG, eletrencefalograma; Pot. Evoc., potencial evocado.

aborto espontâneo. Tinha três irmãos saudáveis - um deles, com história de convulsão febril. Pais eram sadios e não consanguíneos. Mãe negava o uso de drogas potencialmente prejudiciais ao feto durante a gravidez. Nasceu de parto normal, a termo. Foi observada a presença de mecônio no líquido amniótico e teve pneumonia por aspiração que foi tratada com sucesso. Não foram necessárias medidas de suporte respiratório. Havia relato de atraso do desenvolvimento neuropsicomotor: aos 6 meses não sustentava a cabeça, sugava mal e não sorria.

Ao exame, observava-se um lactente com hipomimia facial, em bom estado geral, reagindo com indiferença aos estímulos do meio ambiente. Apresentava estrabismo convergente, mímica facial inexpressiva, epicanto, hipertelorismo ocular, microstomia, mandíbulas hipoplásicas e orelhas de implantação baixa. Havia hipertelorismo mamilar, mancha angiomatosa no antebraço esquerdo e pés tortos congênitos. A avaliação neurológica mostrava hipotonia universal, hiperrreflexia profunda e reflexo cutâneoplantar em extensão. Verificava-se paralisia bilateral do VI e do VII NC. O reflexo cócleo-palpebral estava diminuído, podendo sugerir comprometimento do VIII NC. Havia dúvida quanto ao envolvimento dos IX e X NC (Fig 1).

Os resultados dos exames diagnósticos de investigação estão resumidos na Tabela 1. A tomografia computadorizada do crânio (TCC) sem contraste evidenciava imagens nodulares, isodensas, de tamanhos variados, na região subependimária do ventrículo lateral direito, região hiperdensa no lobo frontal, ectasia ventricular e hipoplasia do vermis cerebelar associada a comunicação direta da cisterna magna com o IV ventrículo - compatível com variante de Dandy Walker. A ressonância magnética cerebral (RMC) (Fig 2) confirmou tais achados, revelando ainda agenesia do vermis cerebelar e hipoplasia do corpo caloso. $\mathrm{O}$ aspecto das nodulações era compatível com o diagnóstico de heterotopia.

\section{DISCUSSÃO}

A SM é de ocorrência rara ${ }^{3,8}$. Entretanto, temos a impressão que vem sendo observada mais frequentemente nos últimos anos. O uso do misoprostol com o objetivo de interromper a gravidez - informação 


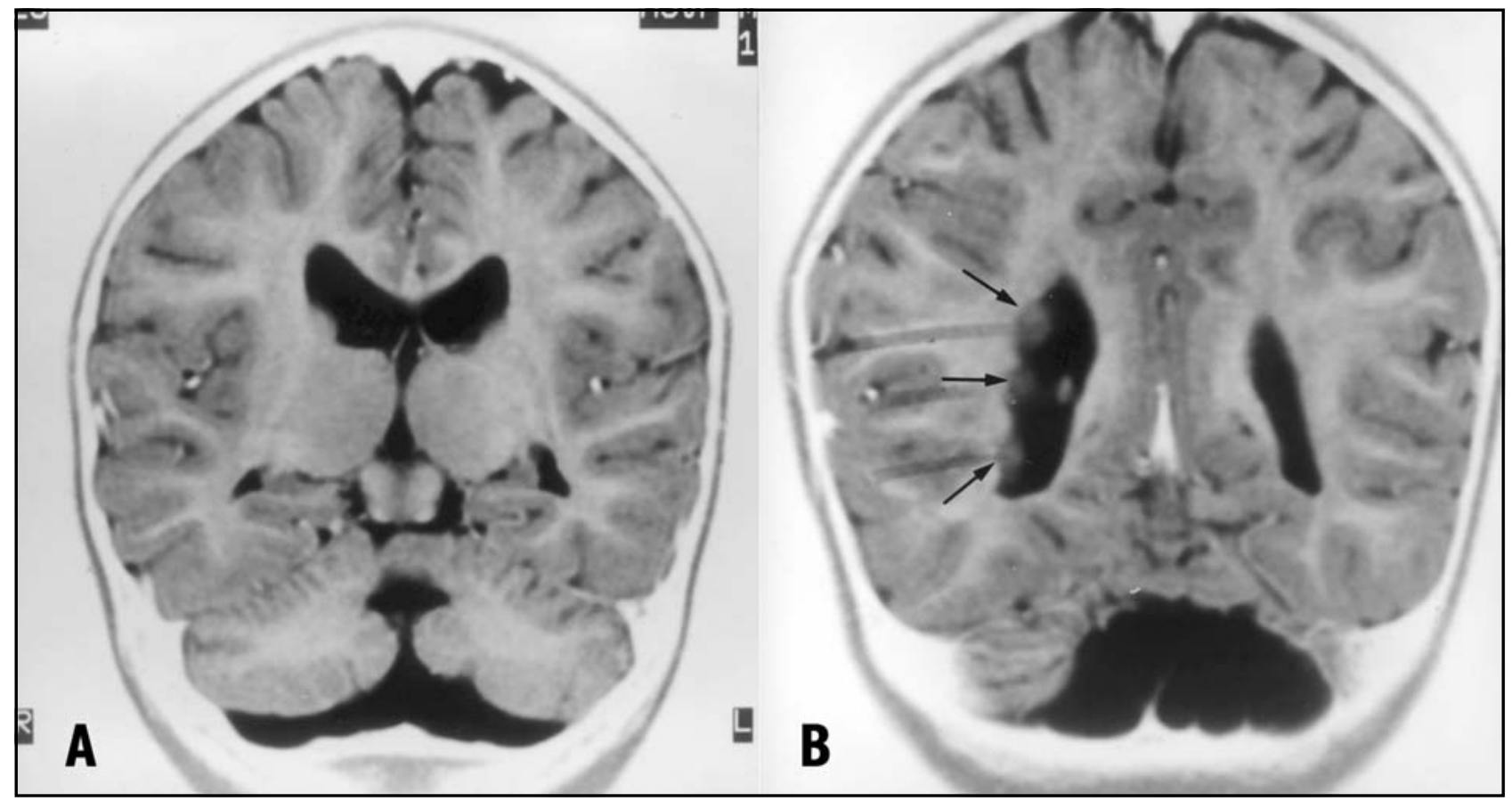

Fig 2. Ressonância magnética cerebral. Observa-se: (a) hipoplasia do corpo caloso; agenesia do vermis cerebelar inferior e megacisterna magna com comunicação direta com o IV ventrículo (variante de Dandy-Walker). (b) formações nodulares subependimárias (setas) sugestivas de heterotopia.

cada vez mais presente na história gestacional de muitas mulheres do nosso meio - deve, na nossa opinião, ter relação com tal fato. A possibilidade de depararmos com maior número de casos da SM talvez crie oportunidades de serem conhecidas outras anormalidades não relacionadas anteriormente ao seu quadro clínico. É possível, também, que os aspectos etiopatogênicos da síndrome possam ser melhor esclarecidos à medida que mais pacientes forem sendo estudados.

O caso relatado mostra sinais clínicos de agenesia ou hipoplasia nuclear de vários nervos cranianos situados no tronco cerebral, próprios da SM, assim como sinais clínicos e radiológicos de outras malformações cerebrais, dentre elas heterotopias nodulares - cuja descrição anterior em associação a essa síndrome desconhecemos. Evento intra-uterino de natureza isquêmica, único e transitório, como defendido pela principal teoria etiopatogênica da SM, segundo nossa avaliação, dificilmente poderia explicar tais achados, uma vez que as malformações visualizadas nas imagens são produzidas em momentos distintos da vida intra-uterina. As heterotopias resultam de migração neuronal desorganizada e arrastada, presumivelmente em torno da $20^{\mathrm{a}}$ semana da gestação ${ }^{9}$ enquanto os núcleos do VII NC, cuja aplasia ou hipoplasia definem a principal característica da SM, diferenciam-se em torno da $5^{\mathrm{a}}$ semana $^{12}$.

\section{REFERÊNCIAS}

1. Moebius P. Ueber angeborene doppelseitige Abducens-FacialisLaehmung. Münch Med Wochenscher 1888;35:91.

2. Marti-Herrero M, Cabrera-Lopez JC, Toledo L, Pérez-Candela V, Bonnet D. Sindrome de Moebius: tres formas diferentes de presentatión. Rev Neurol (Barc) 1998;27:975-978.

3. Rizos M, Negron RJ, Serman N. Möbius syndrome with dental involvement: a case report and literature review. Cleft Palate-Cranialfacial J 1998;35:262-268

4. Matsui A, Nakagawa M, Okuno M. Association of atrial septal defect with Polland Moebius syndrome: vascular disruption. J Vasc Dis 1997;48 269-271.

5. Lahorgue Nunes M, Friedrich MAG, Loch LF. Association of misoprostol, Moebius syndrome and congenital alveolar hypoventilation: case report. Arq Neuropsiquiatr 1999;57:88-91.

6. Fujita I, Koyanagi T, Kukita J, et al. Moebius syndrome with central hypoventilation and braintem calcification: a case report. Eur J Pediatr 1991;150:582-583.

7. Igarashi M, Rose DF, Storgion SA. Moebius syndrome and central respiratory dysfunction. Pediatr Neurol 1997;16:237-240.

8. Dotti MT, Federic Ao, Palmeri S, Guazzi GC. Congenital oculo-facial paralysis (Moebius syndrome): evidence of dominant inheritance in two families. Acta Neurol 1989;11:434-438.

9. Volpe J. Neurology of the newborn. 3.Ed. Philadelphia: Saunders, 1995:43-92.

10. Ziter FA, Wiser WC, RobinsonA. Three generations of a Moebius syndrome variant with chromosome translocation. Arch Neurol 1977;34:437-442.

11. Gonzalez CH, Marques-Dias MJ, Kim CA, Sugayama SMM, Paz JA. Congenital abnormalities in Brazilian children associated with misoprostol misuse in first trimester of pregnancy. Lancet 1998;351: 1624-1627.

12. Gaskill SJ, Marlin AE. Handbook of pediatric neurology and neurosurgery. Boston: Little Brown, 1993: 3-6. 